\title{
Volume kinetics of acetated Ringer's solution during experimental spinal anaesthesia
}

\author{
Robert Hahn, C C Lindahl, and Drobin
}

\section{Linköping University Post Print}

\section{Tweet}

N.B.: When citing this work, cite the original article.

This is the pre-reviewed version of the following article:

Robert Hahn, C C Lindahl, and Drobin, Volume kinetics of acetated Ringer's solution during experimental spinal anaesthesia, 2011, Acta Anaesthesiologica Scandinavica, (55), 8, 987994.

which has been published in final form at:

http://dx.doi.org/10.1111/j.1399-6576.2011.02493.x

Copyright: Wiley-Blackwell

http://eu.wiley.com/WileyCDA/Brand/id-35.html

Postprint available at: Linköping University Electronic Press

http://urn.kb.se/resolve?urn=urn:nbn:se:liu:diva-70736 


\title{
Volume kinetics of acetated Ringer's solution during experimental spinal anesthesia
}

\author{
Robert G. Hahn, ${ }^{1,2}$ Christina C. Lindahl ${ }^{3}$, and Dan Drobin ${ }^{1}$ \\ ${ }^{1}$ Department of Anesthesia at the Faculty of Health Sciences, Linköping University, \\ Linköping, ${ }^{2}$ Research Unit, Södertälje Hospital, Södertälje, Sweden. ${ }^{3}$ Department of \\ Anesthesia, Karolinska University Hospital, Stockholm,
}

The study was conducted at Södersjukhuset, Stockholm, Sweden, where all authors worked when the data was collected.

Address for correspondence:

Robert G. Hahn, MD, PhD

Section of Anesthesia

Faculty of Health Sciences

Linköping University

58185 Linköping, Sweden

Telephone: +467395660972

E-m ail: $\underline{\text { r.hahn } @ \text { @telia.com }}$

Short title: Fluid dynamics during spinal anesthesia 


\section{Abstract}

Background: General anesthesia lowers the clearance of crystalloid fluid, but the volume kinetics of such fluid throughout the duration of spinal anesthesia has not been studied.

Methods: Ten female volunteers (mean age 29 years) received an intravenous infusion of $25 \mathrm{ml} / \mathrm{kg}$ of acetated Ringer's solution with and without spinal anesthesia. A volume kinetic model was fitted to serial measurements of the hemoglobin concentration over 240 min based on arterial, cubital vein, and femoral vein blood. The measured urine flow was compared to the model-predicted elimination.

Results: The arterial pressure remained stable although the block reached to Th3-Th5 in half of the volunteers. There were no differences in fluid kinetics between the spinal anesthesia and the control experiments. The administered volume was well confined to the kinetic system, which consisted of two communicating fluid spaces that were $2.8 \mathrm{~L}$ and approximately $7 \mathrm{~L}$ in size at baseline. The arteriovenous (AV) difference in plasma dilution remained positive for $30 \mathrm{~min}$ post-infusion in those having analgesia reaching to Th3-Th5, which differed significantly from low-level analgesia (Th12-L2, $P<0.03)$ when venous plasma was sampled from the leg. The urinary excretion averaged $1.13 \mathrm{~L}$ and $1.01 \mathrm{~L}$ for the spinal and control experiments, respectively. Volume kinetics predicted the urinary excretion at 5-10 min intervals with an overall bias of $52 \mathrm{ml}$.

Conclusion: Acetated Ringer's solution showed the same kinetics during experimental spinal anesthesia as when the fluid was infused alone. Hence, spinal anesthesia is not associated with the reduced fluid clearance reported for general anesthesia.

Key words. Anesthetic techniques, subarachnoid;

Fluids, IVi.v.; Model, pharmacokinetic; 


\section{Introduction}

Intravenous (IV) fluid therapy remains the first choice for cardiovascular support during anesthesia and surgery. Volume kinetic studies show that the renal clearance $\left(C l_{\mathrm{r}}\right)$ for crystalloid fluid is greatly reduced during general anesthesia. During thyroid ${ }^{1}$ as well as laparoscopic ${ }^{2}$ and open abdominal ${ }^{3}$ surgery, $C l_{\mathrm{r}}$ is only $5-20 \mathrm{ml} / \mathrm{min}$, while it is usually $60-100 \mathrm{ml} / \mathrm{min}$ in conscious volunteers. ${ }^{4,5}$ Isoflurane alone lowers $C l_{\mathrm{r}}$ for crystalloid fluid by $50 \% .{ }^{6}$ The distribution of fluid may also become aberrant. ${ }^{1,7}$

In contrast to general anesthesia, the capacity of spinal anesthesia to modify the kinetics of crystalloid fluid is poorly known. Just as with general anesthesia, ${ }^{8}$ the onset of spinal anesthesia lowers the distribution clearance $\left(C l_{\mathrm{d}}\right)$ in proportion to the reduction of the arterial pressure. ${ }^{8-10}$ However, the distribution and elimination of crystalloid fluid throughout the course of spinal anesthesia has not been studied.

The aim of the present study was to compare the kinetics of acetated Ringer's solution with and without experimental spinal anesthesia in volunteers. The distribution of fluid in the presence of spinal block was further explored by collecting blood from three sampling sites and by continuous collection of excreted urine. Our hypothesis was that spinal anesthesia increases the plasma volume expansion in response to crystalloid fluid either by reducing $C l_{\mathrm{d}}$ or $C l_{\mathrm{r}}$. We also wished to examine whether the anesthesia promotes aberrant distribution of the administered fluid. 


\section{Materials and Methods}

Ten healthy female volunteers, aged 21-39 (mean 29) years with a body weight of 58$67 \mathrm{~kg}$ (mean $62.5 \mathrm{~kg}$ ) participated as paid volunteers in two experiments separated by at least one week. The protocol was approved by the Regional Ethics Committee of Karolinska institutet. Each volunteer gave her written informed consent after a medical examination confirmed that she was in good health. None of them used daily medication.

The volunteers arrived at the hospital at 8 AM. No oral fluid or food was allowed between midnight and completion of the experiment. Both experiments consisted of the subject receiving an IV infusion of $25 \mathrm{ml} / \mathrm{kg}$ acetated Ringer's solution ( $\mathrm{Na} 130, \mathrm{Cl} 110, \mathrm{Ca} 2, \mathrm{~K} \mathrm{4}$, acetate- $30 \mathrm{mmol} / \mathrm{L}$; osmolality $273 \mathrm{mosm} / \mathrm{L}$ ) provided by an infusion pump (Flo-Guard 6201, Baxter, IL). Blood and urine were sampled over 240 min for later kinetic calculations of the distribution and elimination of the volume load.

On one occasion, the volunteers received fluid and an experimental spinal anesthesia while on the other occasion they received only the fluid volume (control).

\section{Experimental procedure}

Instrumentation during the spinal anesthesia experiment consisted of cannule for sampling blood from the radial artery, cubital vein, and femoral vein, and placement of an indwelling catheter into the bladder. A cannula was also placed in the cubital vein of the opposite arm for the administration of fluid. A period of $20 \mathrm{~min}$ in the supine position was allowed for equilibration before the infusion was started, and the volunteers remained in bed during the observation time.

Preloading was performed as a safeguard against undetected hypovolemia. Hence, acetated Ringer's solution was infused over $60 \mathrm{~min}$ and spinal anesthesia induced after $30 \mathrm{~min}$. For this purpose, the volunteer was placed in a lateral position until the anesthesia was given, after which the supine position reassumed. We used a Whitacre pencil-point 26 gauge needle (Becton Dickinson, Franklin Lake, NJ) and 3.0 
$\mathrm{ml}$ bupivacaine hydrochloride $5 \mathrm{mg} / \mathrm{ml}$ (AstraZeneca AB, Södertalje, Sweden) was administered medially in the lumbar 3-4 interspace. The spread of the analgesia was tested with loss of cold sensation every 5-10 min until the block began to subside. Intravenous ephedrine 5-10 mg was planned to be withheld until arterial hypotension coincided with nausea and/or bradycardia.

The control experiment differed from the first protocol in that blood was only sampled from a cubital vein and that the acetated Ringer's solution was administered as a standard 30-min infusion. ${ }^{4,5,11}$

\section{Measurements}

Blood samples $(4 \mathrm{ml})$ were taken, and the urinary excretion was measured every $5 \mathrm{~min}$ during the first $120 \mathrm{~min}$ and every $10 \mathrm{~min}$ thereafter up to $240 \mathrm{~min}$. A discard volume of $3 \mathrm{ml}$ was drawn before each blood collection. This blood was then returned and the cannula flushed with $3 \mathrm{ml}$ of saline to prevent clotting and to replace the amount of withdrawn plasma.

The hemoglobin $(\mathrm{Hb})$ concentration in whole blood, the red blood cell count, and the mean corpuscular volume were measured by a Technicon H2 (Bayer, Tarrytown, NY) using colorimetry at $546 \mathrm{~nm}$ for $\mathrm{Hb}$, and light dispersion using a helium neon laser for the other two parameters. Duplicate samples drawn at baseline assured a $\mathrm{CV}$ of $0.8 \%$ for $\mathrm{Hb}(\mathrm{n}=40)$; the mean values were used in the calculations.

Heart rate and non-invasive arterial pressure were displayed on an AS 3monitor (Datex, Finland). Peripheral oxygen saturation and EKG were also monitored. Each experiment was performed by two anesthesiologists and one anesthesia nurse, which allowed withdrawal of exactly timed blood samples and assured the best possible control of the safety of the volunteers.

\section{Kinetic analysis}

The distribution and elimination of the fluid given by IV infusion was analyzed by fitting a two-volume fluid-space model to the $\mathrm{Hb}$ derived plasma dilution..$^{1-8,11}$ A clearance constant $C l$ describes elimination, $C l_{\mathrm{d}}$ describes distribution and $C l_{\mathrm{o}}$ denotes baseline fluid losses (pre-set to 0.4 $\mathrm{ml} / \mathrm{min}^{13}$ ). The infusion is given at rate $R_{\mathrm{o}}$ and expands two functional volumes, a 
central volume $V_{\mathrm{c}}$ to the larger size $v_{\mathrm{c}}$ and a peripheral volume $V_{\mathrm{t}}$ to $v_{\mathrm{t}}($ Fig. 1$)$. The differential equations are:

$$
\begin{aligned}
& \frac{d \nu_{\mathrm{c}}}{d t}=R o-C l_{\mathrm{o}}-C l \frac{\left(\nu_{\mathrm{c}}(t)-V_{\mathrm{c}}\right)}{V_{\mathrm{c}}}-C l_{\mathrm{d}}\left[\frac{\left(\nu_{\mathrm{c}}(t)-V_{\mathrm{c}}\right)}{V_{\mathrm{c}}}-\frac{\left(\nu_{\mathrm{t}}(t)-V_{\mathrm{t}}\right)}{V_{\mathrm{t}}}\right] \\
& \frac{d \nu_{\mathrm{t}}}{d t}=C l_{\mathrm{d}}\left[\frac{\left(\nu_{\mathrm{c}}(t)-V_{\mathrm{c}}\right)}{V_{\mathrm{c}}}-\frac{\left(\nu_{\mathrm{t}}(t)-V_{\mathrm{t}}\right)}{V_{\mathrm{t}}}\right]
\end{aligned}
$$

at any time $t$. The Hb-derived fractional plasma dilution was used to indicate the volume expansion of $V_{\mathrm{c}}$ resulting from the in fu sion:

$$
\frac{v_{\mathrm{c}}(t)-V_{\mathrm{c}}}{V_{\mathrm{c}}}=\frac{[\mathrm{Hb} / \mathrm{Hb}(t)]-1)}{(1-\mathrm{Hct})}
$$

A correction for the effect of blood sampling was made on the plasm a dilution. ${ }^{1}$

The renal clearance of the infused fluid $\left(\mathrm{Cl}_{r}\right)$ was calculated based on the total urinary excretion divided by the area under the curve (AUC) of the plasma dilutiontime profile: ${ }^{4}$

$$
C l_{r}=\frac{\sum \text { urine volume }}{A U C \text { for }\left(v_{\mathrm{c}}(t)-V_{\mathrm{c}}\right) / V_{\mathrm{c}}}
$$

The matrix solutions to the differential equations describing the two-volume kinetic model ${ }^{4}$ were fitted to the data from each volunteer and sampling site separately by using a nonlinear least-squares regression routine, based on a Gauss-Newton method and programmed in Matlab 4.2 (MathWorks Inc., Natick, MA). This was repeated until no parameter changed by more than $0.1 \%$ in each iteration.

The volumes of $v_{\mathrm{c}}$ and $v_{\mathrm{t}}$ over time were calculated by multiplying their dilutions with $V_{\mathrm{c}}$ and $V_{\mathrm{t}}$, which transformed $\left(v_{\mathrm{c}}-V_{\mathrm{c}}\right) / V_{\mathrm{c}}$ into $\left(v_{\mathrm{c}}-V_{\mathrm{c}}\right)$. 
The urinary excretion over time was assessed in three ways: (1) from the urine collections at 5-10 min intervals, (2) as the cumulative product of the plasma dilution at any time and the $\mathrm{Cl}$ obtained by the curve-fitting, and (3) as the cumulative product of the plasma dilution and $C l_{\mathrm{r}}$, the latter of which was based on the total urinary excretion between 0 and $240 \mathrm{~min}$. 


\section{Statistics}

Data are presented as the mean and standard deviation (SD) or, if the distribution was skewed, as the median and interquartile range. Statistics was performed by using oneway and repeated-measures ANOVA, the Wilcoxon matched-pair test, and linear regression analysis. A systematic difference between predicted and measured data (i.e. the bias) was expressed as the median residual error. The absolute value for the difference between predicted and measured data was used to quantify the precision of the prediction. $P<0.05$ was considered statistically significant.

\section{Results}

\section{Spinal block and hemodynamics}

After induction at $30 \mathrm{~min}$, the onset of the block was rapid and the cephalic spread peaked at 60 (51-65) min. The upper level of the analgesia reached Th5 (Th3-L2) and the block began to subside at 108 (101-122) min (Fig. 2 A). It was not possible to safely determine the return of sensation due to patchy recovery, but half of the volunteers had residual analgesia at the end of the experiment (at $240 \mathrm{~min}$ ).

The arterial pressure tended to be slightly higher at baseline when spinal anesthesia was to be induced, but the difference from the controls was not statistically significant (Table 1). Minor hemodynamic changes occurred in the presence of spinal anesthesia (Fig. 2 B-D). Here, the arterial pressure declined slightly, the lowest being recorded at $60 \mathrm{~min}$ (30 $\mathrm{min}$ after the induction).

One volunteer was given $10 \mathrm{mg}$ ephedrine intravenously when her systolic arterial pressure fell from 113 to $79 \mathrm{mmHg}$ in $10 \mathrm{~min}$, after which the pressure recovered instantly to $104 \mathrm{mmHg}$. This was the only complication. The event occurred in the volunteer who had the fastest onset of the anesthesia but was not associated with nausea or bradycardia. 


\section{Plasma dilution}

The induction of anesthesia created a temporary notch in the plasma dilution-time profile, but there was no marked difference in its overall shape as compared to the control infusions (Fig. 3).

As the dilution-time curves from the three sampling sites had quite similar appearance (Fig. 3 A-C), the distribution of fluid was further explored by plotting the AV differences in plasma dilution (Fig. 3 D, E). The arm showed a slightly greater AV difference than the leg, but statistical significance was not reached. However, the six volunteers with the highest level of analgesia (Th3-Th5) had a positive AV difference for some time even after the infusion ended, which was not the case for the four having low-level analgesia (Th12-L2). This difference between low-level and highlevel analgesia was most apparent in the leg at between 65-90 min of the study, where it also reached statistical significance $(P<0.03)$.

Arterial blood tended to yield lower $V_{\mathrm{c}}$ than venous blood, but otherwise kinetic parameter estimates from the three sampling sites were quite similar (Table 2). The renal clearance $\left(C l_{\mathrm{r}}\right)$ was close that the total clearance $(C l)$.

There was no significant difference between the two series of experiments with regard to the fluid kinetics calculated from cubital vein blood (Table 2).

\section{Simulations}

The median values of the parameter estimates based on cubital vein blood were used for further computer simulations to highlight the disposition of the infused fluid.

The fluid volume residing in $v_{\mathrm{t}}$ already exceeded that of $v_{\mathrm{c}}$ at the end of the infusions. At that time, $24 \%$ and $40 \%$, respectively, of the fluid administered during the spinal and control experiments, respectively, remained in $v_{\mathrm{c}}$ (Fig. $4 \mathrm{~A}, \mathrm{~B}$ ). This difference was due to the infusion time, as the fraction of the fluid that was retained in $v_{\mathrm{c}}$ decreased in a similar way throughout the infusion period (Fig. $4 \mathrm{C}$ ).

The relationship between the excess volumes residing in $V_{\mathrm{t}}$ and $V_{\mathrm{c}}$ [i.e. the ratio $\left.\left(v_{\mathrm{t}}-V_{\mathrm{t}}\right) /\left(v_{\mathrm{c}}-V_{\mathrm{c}}\right)\right]$ stabilized $25 \mathrm{~min}$ after each infusion at a ratio of approximately 3 (Fig. 4 D). This ratio was slightly higher than the relationship between the baseline volumes $V_{\mathrm{t}} / V_{\mathrm{c}}$ due to the fact that elimination occurs from $v_{\mathrm{c}}$ (Table 2). 


\section{Urinary excretion}

The total urinary excretion was similar for the two experiments (Table 1, bottom). The urine flow was assessed by frequent direct measurement but also by two kinetic approaches (Fig. 5 A-D). A comparison shows that the two kinetic approaches slightly overestimated the urinary excretion, the overall median residual error (the bias) being $52 \mathrm{ml}$. The median absolute residual error (the inaccuracy) for the difference between the predicted and measured urine volumes based on all data points for both kinetic methods was $69 \mathrm{ml}$ (Fig. 5 E, F). There was a strong overall linear correlation between the clearance values obtained by the two kinetic approaches (Fig. 6).

\section{Discussion}

The distribution and elimination of acetated Ringer's solution during experimental spinal anesthesia was quite similar to that of a control infusion. Hence, the results refute our hypothesis that pronounced plasma volume expansion would result from crystalloid fluid during spinal anesthesia alone. We believed that the mechanism for such a change could be a reduction of either $C l_{\mathrm{d}}$ or $C l_{\mathrm{r}}$, but no such change occurred.

Previous work shows that the onset on both general an regional anesthesia markedly reduces $C l_{\mathrm{d}}$ which degree correlates with the accompanying decrease in arterial pressure. ${ }^{8-10,12}$ The fact that the $C l_{\mathrm{d}}$ reported here was close to values previously found in volunteers not receiving anesthesia ${ }^{4,11}$ can be understood from the stable arterial pressure recorded in nearly all of the present subjects (Fig. 2). Hence, spinal block without hypotension does not change the fluid distribution.

The fluid clearance, $C l$, was slightly above $100 \mathrm{ml} / \mathrm{min}$, which is normal for volunteers, ${ }^{14}$ although lower values have occasionally been reported. ${ }^{5}$ In any event, the very marked reduction of $\mathrm{Cl}$ to only $5-20 \mathrm{ml} / \mathrm{min}$ during thyroid ${ }^{1}$ and abdominal surgery $^{2,3}$ and general anesthesia alone ${ }^{6}$ was not at hand. The $C l$ rather tended to be slightly higher with than without the block (Table 2). 
Further calculations challenged whether all elimination could be attributed to renal excretion. This proved to be the case, as the renal clearance, $C l_{\mathrm{r}}$, differed only a few percent from $\mathrm{Cl}$ based on blood samples only (Table 2, Fig. 6). Hence, all administered fluid could be accounted for in the kinetic system, and there was no evidence of aberrant distribution or peripheral accumulation of fluid.

The kinetic analysis is based on the concept that infused fluid is first distributed in a central fluid space, $V_{\mathrm{c}}$. Using data from venous plasma, $V_{\mathrm{c}}$ had a size virtually identical to the expected size of the plasma volume $(4.5 \%$ of the body weight). The size of the fluid space to which the fluid further distributed, $V_{\mathrm{t}}$, was 2-3 times larger. As in previous work, ${ }^{1,4} 5 V_{\mathrm{t}}$ was still smaller than the size of the interstitial fluid space as it corresponded to $12 \%$ of the body weight instead of the expected $15 \%$. This difference is probably due to that parts of the interstitial fluid space have a low compliance for volume expansion. ${ }^{14}$

The size of $V_{\mathrm{c}}$ tended to be slightly smaller when based on arterial as compared to venous blood. ${ }^{15}$ This is the result of a slightly more pronounced dilution of arterial plasma during the infusion, which is to be expected, as some of the ongoing infused volume is sampled before having passed the capillaries for the first time. When an infusion of Ringer's is turned off, the AV difference for plasma dilution in the arm becomes reversed after 2.5 min. ${ }^{15}$ In the present study, the reversal occurred within at least $5 \mathrm{~min}$ in volunteers with low-level analgesia. However, those having the upper level of analgesia reaching to Th3-Th5 continued to show a positive AV difference until the block began to subside, which means that fluid was transported from blood to tissue throughout the period of maximum analgesia. This effect of high-level analgesia was slightly more apparent in the leg, where the difference from low-level analgesia even reached statistical significance.

The present study also contained the first evaluation of how well urinary excretion is predicted by the kinetic model throughout an infusion experiment. Urine was collected frequently and compared to the model-predicted elimination based on $\mathrm{Cl}$ (from blood samples) as well as on $C l_{\mathrm{r}}$ (from total urine volume and blood samples). The capacity of the conventional two-volume kinetic model to predict urinary excretion must be considered to be good (Fig. 5). The model-based curves were often placed slightly higher than the measured urinary excretion in Fig. 5 E-F, which is probably due to the fact that some time is needed for the urine to pass through the 
kidneys and ureters in order to enter an external urine bag. This delay affected the urine collections at all times.

Limitations of the study include that safety reasons prompted a 30-min preloading period before spinal anesthesia was induced, which is included in the kinetic analysis. The block resolved at various speeds, whereby some late periods of limited anesthesia could be included in the kinetic analysis. Besides that the volume loading before the block is induced is still widely practiced clinically, partial derivatives show that the data obtained during the period when the anesthesia was fully developed contributed the most information to the parameter estimates.

Consideration should be given to the fact that the frequent blood sampling from three sites in the spinal anesthesia experiment created a more or less continuous small bleeding which finally amounted to $400 \mathrm{ml}$. The plasma was replaced by crystalloid fluid, but the loss of $\mathrm{Hb}$ was corrected for in our computer program, as it creates iatrogenic dilution. ${ }^{1}$ Without such a correction, the return of the hemodilution would not be as prompt as shown in Fig. 3. In contrast, the collected blood during the control experiments amounted to only slightly below $150 \mathrm{ml}$.

In conclusion, the volume kinetics of acetated Ringer's solution during experimental spinal anesthesia with stable hemodynamics did not differ from that obtained for a control infusion. In both experiments the urinary excretion was well predicted by the kinetic analysis.

Acknowledgement: Eva Thuresson assisted during the experiments. Financial support was given by Södersjukhuset AB Research Fund. 


\section{References}

1. Ewaldsson C-A, Hahn RG. Kinetics and extravascular retention of acetated Ringer's solution during isoflurane and propofol anesthesia for thyroid surgery. Anesthesiology 2005; 103: 460-9

2. Olsson J, Svensén CH, Hahn RG. The volume kinetics of acetated Ringer's solution during laparoscopic cholecystectomy. Anesth Analg 2004; 99: 1854-60

3. Svensén CH, Olsson J, Hahn RG. Intravascular fluid administration and hemodynamic performance during open abdominal surgery. Anesth Analg 2006: 103: $671-6$

4. Hahn RG, Drobin D. Urinary excretion as an input variable in volume kinetic analysis of Ringer's solution. Br J Anaesth 1998; 80: 183-8

5. Drobin D, Hahn RG. Kinetics of isotonic and hypertonic plasma volume expanders. Anesthesiology 2002; 96: 1371-80

6. Norberg Å, Hahn RG, Husong Li, Olsson J, Prough DS, Börsheim E, Wolf S, Minton R, Svensén CH. Population volume kinetics predicts retention of $0.9 \%$ saline infused in awake and isoflurane-anesthetized volunteers. Anesthesiology 2007; 107: 24-32

7. Connolly CM, Kramer GC, Hahn RG, Chaisson NF, Svensén C, Kirschner RA, Hastings DA, Chinkes D, Prough DS. Isoflurane but not mechanical ventilation promotes extravascular fluid accumulation during crystalloid volume loading. Anesthesiology 2003; 98: 670-81

8. Li Y, Zhu S, Hahn RG. The kinetics of Ringer's solution in young and elderly patients during induction of general and epidural anesthesia. Acta Anaesth Scand 2007; 51: 880-7

9. Drobin D, Hahn RG. Time course of increased haemocdilution in hypotension induced by extradural anaesthesia. Br J Anaesth 1996: 77: 223-6

10. Ewaldsson C-A, Hahn RG. Volume kinetics during induction of spinal and general anaesthesia. Br J Anaesth 2001; 87: 406-14 
11. Drobin D, Hahn RG. Volume kinetics of Ringer's solution in hypovolemic volunteers. Anesthesiology 1999; 90: 81-91

12. Hahn RG. Volume kinetics for infusion fluids (review). Anesthesiology 2010; 113: 470-81

13. Cox P. Insensible water loss and its assessment in adult patients: A review. Acta Anaesthesiol Scand 1987; 31: 771-6

14. Aukland K, Reed RK. Interstitial-lymphatic mechanisms in the control of extracellular fluid volume. Physiol Rev 1993; 73: 1-78

15. Svensén CH, Rodhe PM, Olsson J, Borsheim E, Aarsland A, Hahn RG. Arteriovenous differences in plasma dilution and the distribution kinetics of lactated Ringer's solution. Anesth Analg 2009; 108: 128-33 


\section{Table 1}

Baseline values for blood pressure and heart rate, and key data on fluid balance

Variable

Spinal anesthesia

Control experiment

Systolic arterial pressure $(\mathrm{mm} \mathrm{Hg})$

$115(11)$

$109(10)$

Diastolic arterial pressure $(\mathrm{mm} \mathrm{Hg})$

$70(8)$

$67(7)$

Heart rate (bpm)

$70(13)$

68 (14)

Infused volume (ml)

1463 (233)

$1466(143)$

Urinary excretion (ml)

1133 (400)

1013 (466)

Excreted/Infused (ratio)

$0.77(0.22)$

$0.70(0.32)$

Data are the mean (SD) for the group of 10 volunteers. 


\section{Table 2}

Top: Optimal parameter estimates for kinetic analysis according to the two-volume model, where baseline fluid losses are considered by setting $C l_{\mathrm{o}}$ to $0.4 \mathrm{ml} / \mathrm{min}$.

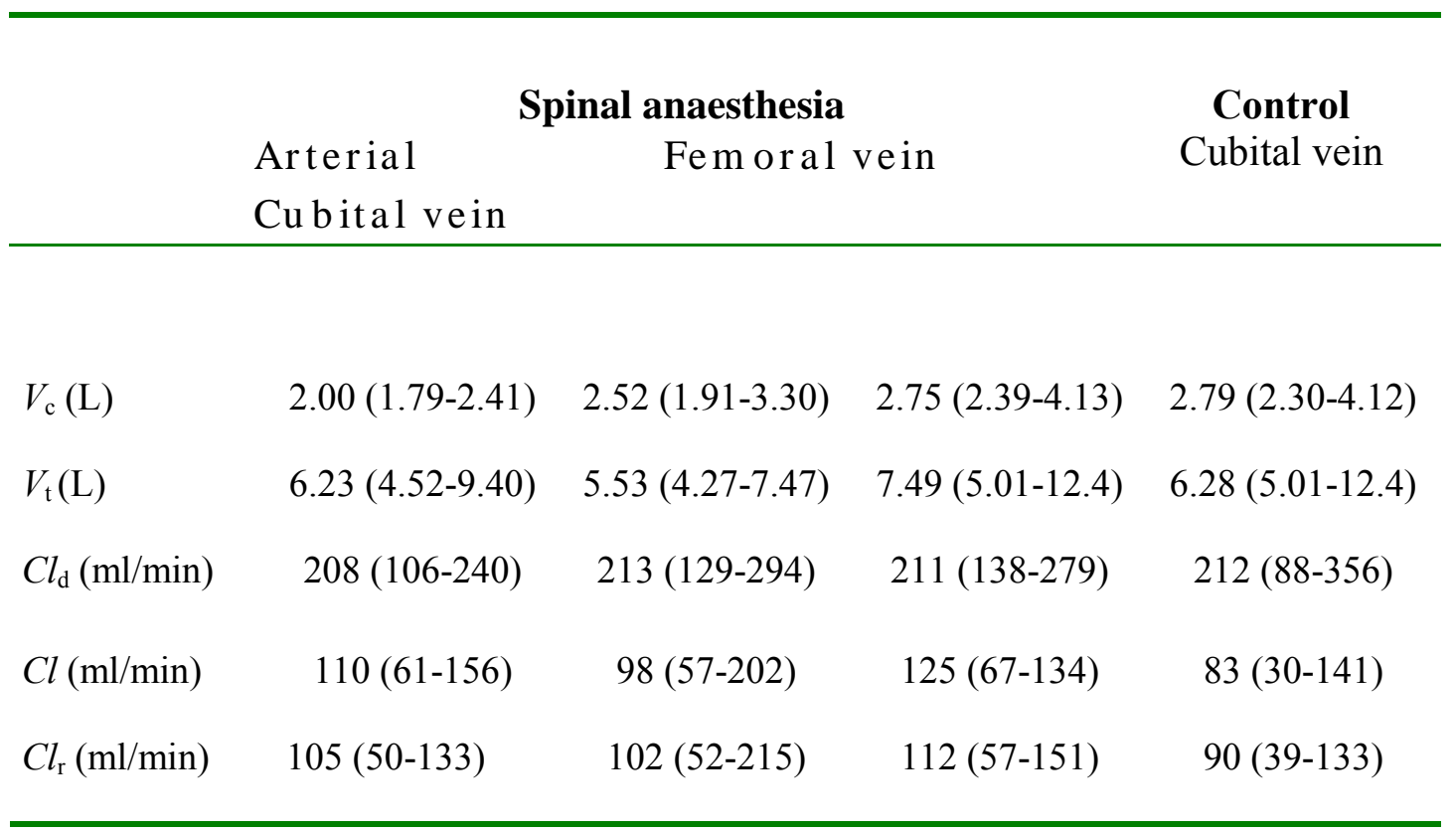

Data are the median (interquartile range) for the group of 10 volunteers.

The uncertainty of an individual parameter estimate, expressed as the SD, averaged $13 \%$ for $\mathrm{Cl}$. 


\title{
Legends for figures
}

\section{Fig. 1}

The two-volume kinetic model used to analyze the data.

Symbols denote: $R_{\mathrm{o}}=$ fluid infusion; $V_{\mathrm{c}}$ and $V_{\mathrm{t}}=$ central and peripheral body fluid spaces, respectively; $v_{\mathrm{c}}$ and $v_{\mathrm{t}}=$ expanded fluid spaces; $\mathrm{Cl}=$ elimination clearance; $C l_{\mathrm{d}}=$ distribution clearance; $C l_{\mathrm{o}}=$ baseline fluid losses.

\section{Fig. 2}

\begin{abstract}
A. Upper level of sensory loss, B. systolic arterial pressure, C. diastolic arterial pressure, and D. heart rate when spinal anesthesia was induced after $30 \mathrm{~min}$. Each line represents one volunteer. In panels B-D the mean in marked by a thick line.
\end{abstract}

\section{Fig. 3}

The plasma dilution during experimental spinal anesthesia indicated in blood from
A. the radial artery,
B. the cubital vein,
C. the femoral vein, D. the AV difference as obtained from the arm, and E. the leg. F. The plasma dilution in the cubital vein measured during a control in fusion. 
Each thin line represents one volunteer. The thick lines in A-C and F are kinetic curves based on the median optimal estimates of the parameters for the group. The thick lines in D and E are the mean values.

Fig. 4

The volume changes of $v_{\mathrm{c}}$ and $v_{\mathrm{t}} \mathrm{during}$ and after an infusion of $25 \mathrm{ml} / \mathrm{kg}$ of acetated Ringer's solution is in fused:

A. during $60 \mathrm{~min}$ in the presence of spinal anesthesia

B. during 30 min without spinal anesthesia (control)

C. the fraction of the infused fluid volume remaining in $v_{c}$, and

D. the ratio between the volume changes of $v_{\mathrm{t}}$ and $v_{\mathrm{c}}$

\section{Fig. 5}

The urinary excretion:

A. measured at 5-10 min intervals during spinal anesthesia

B. predicted by kinetic analysis of venous plasma.

C. predicted from the renal clearance, which is based on the total urine excretion

D. measured at 5-10 min intervals during a control infusion. Each thin line represents one volunteer and the thick line the mean value 
E and F. given as the mean values for the three ways to assess urinary excretion for the spinal and control experiments, respectively

Fig. 6

The fluid clearance, $C l$, estimated by curve-fitting of the plasma dilution over time versus the renal clearance, $C l_{\mathrm{r}}$, which was calculated as the measured total urinary excretion divided by the area under the curve of the plasma dilution-time profile. 


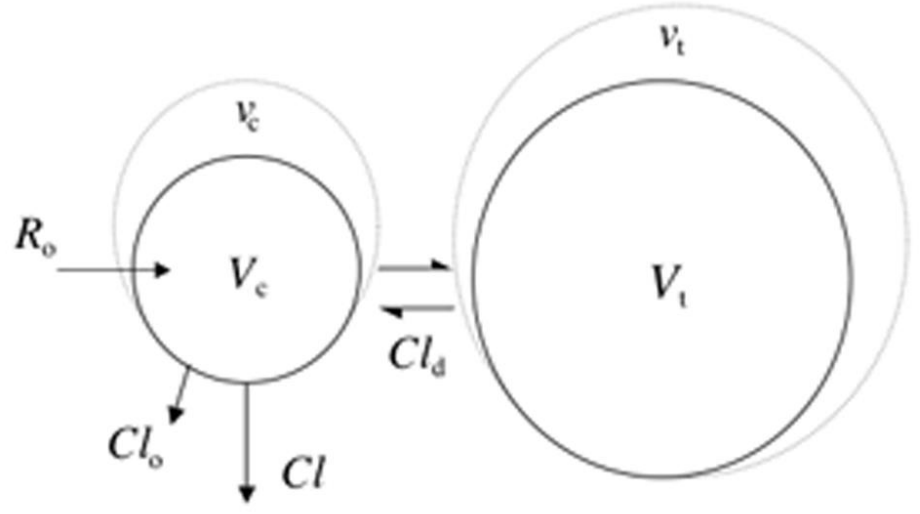

Fig. 1 

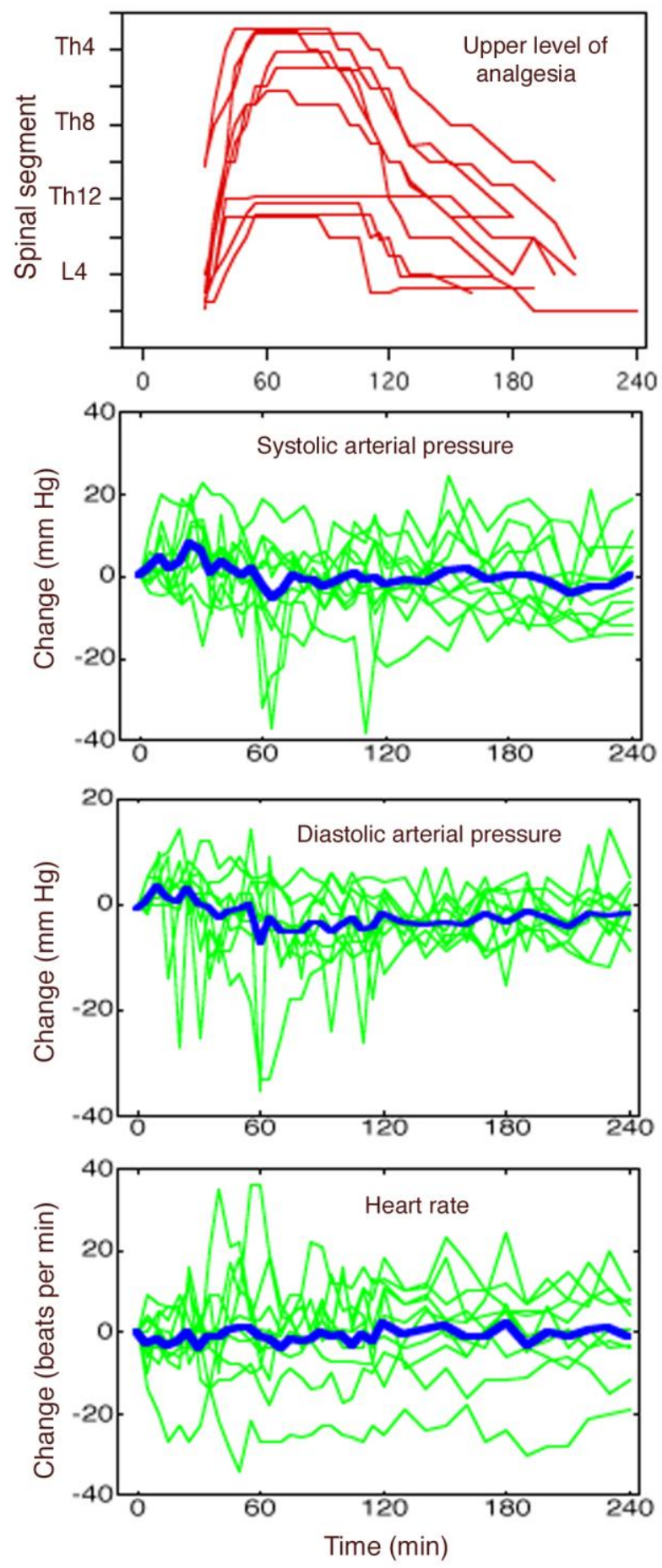

Fig. 2 

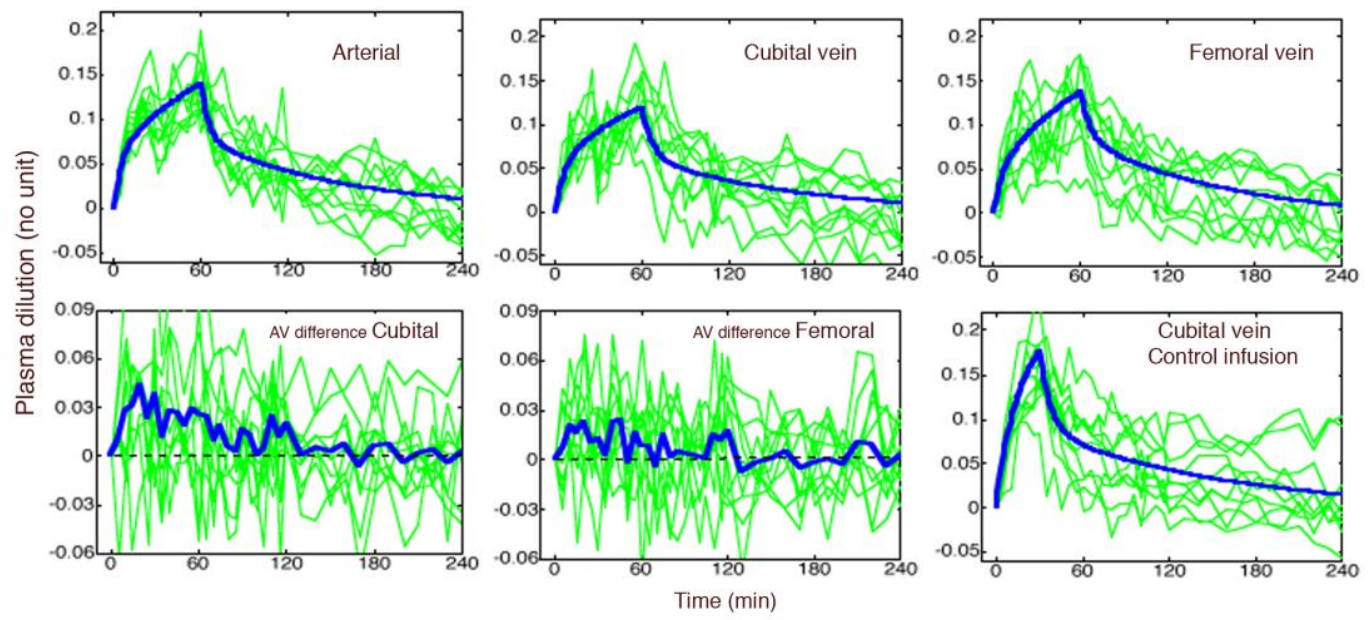

Fig. 3
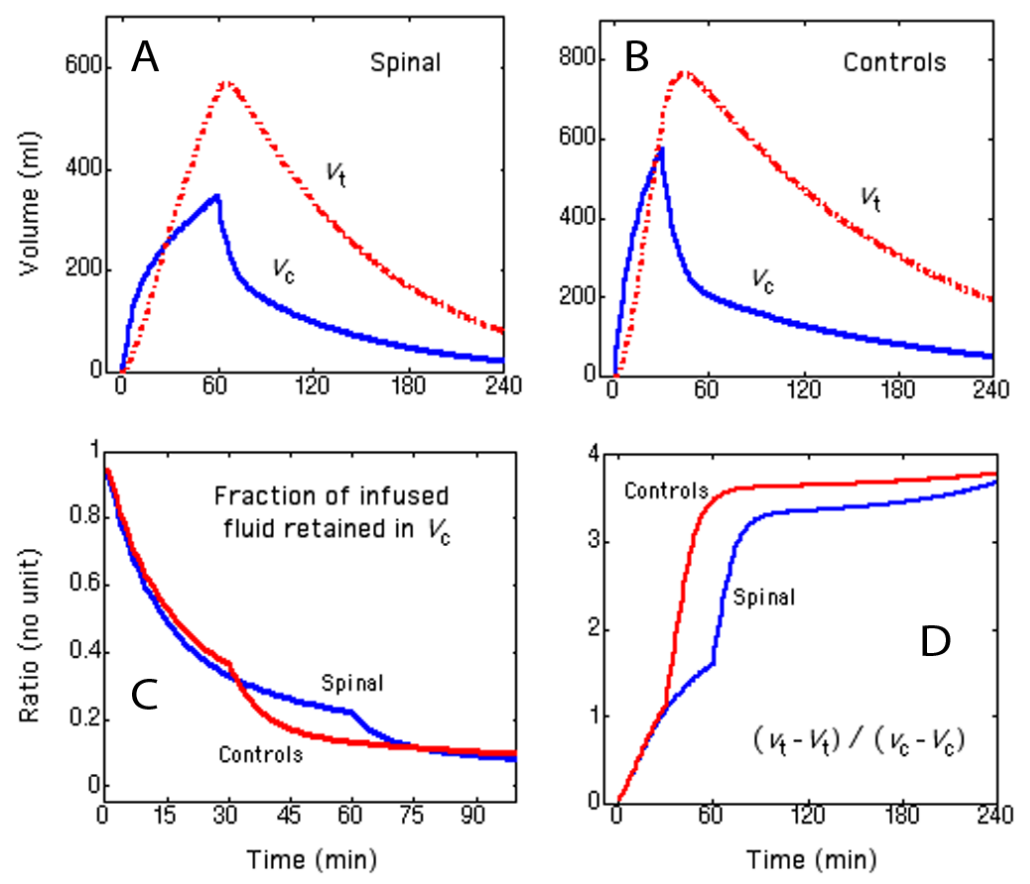

Fig. 4 

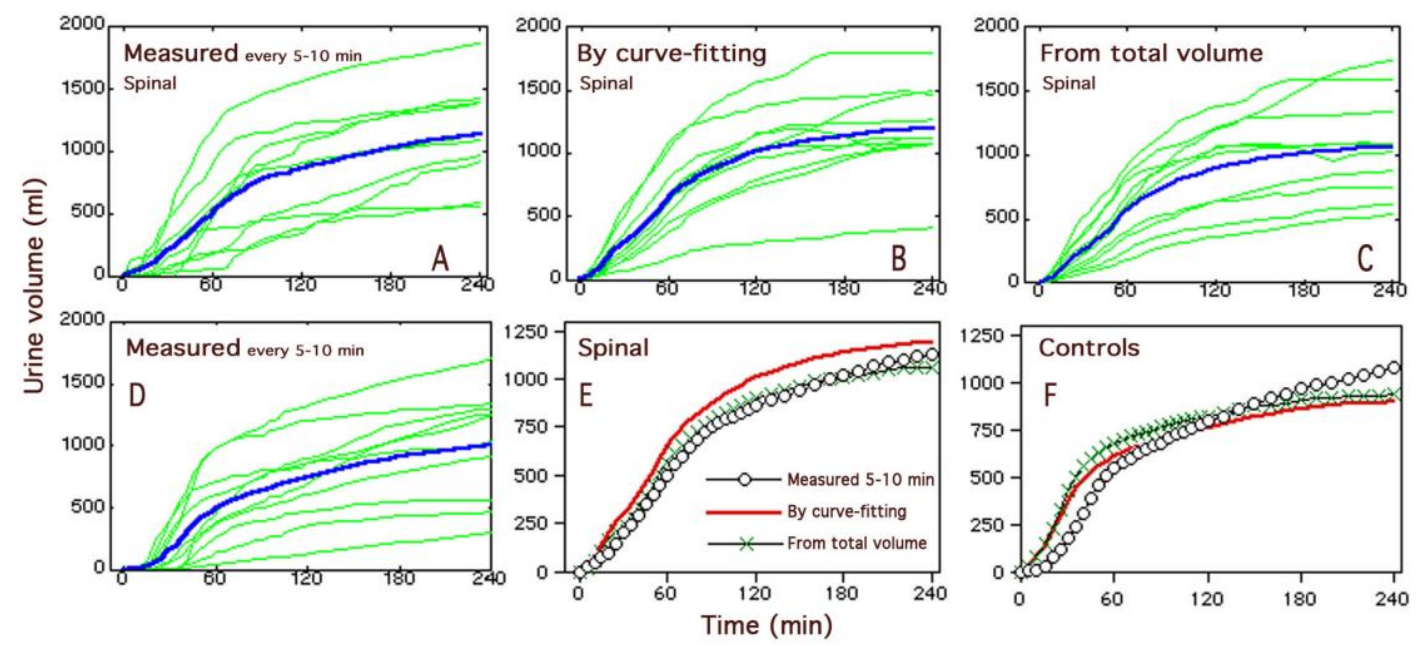

Fig. 5

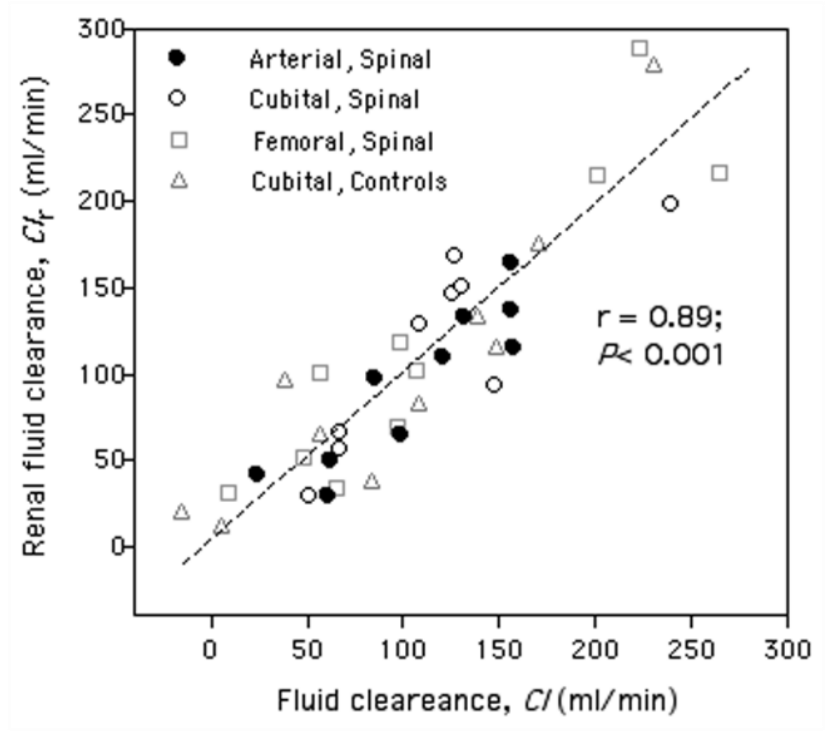

Fig. 6 